\title{
Riboflavin Mediated Degradation of Chlorpyrifos by Alcaligens sp. Strain T11
}

\author{
Saira Khalid ${ }^{1}$, and Imran Hashmi ${ }^{1}$
}

\begin{abstract}
Chlorpyrifos (CP) is known to be toxic for all living organisms. Increasing pesticide concentration in environment as a result of agricultural and industrial runoff has attained attention in recent years. Bacterial strain capable of $\mathrm{CP}$ biodegradation was isolated from pesticide contaminated soil and identified using $16 \mathrm{~S}$ rRNA analysis as Alcaligens sp. T11. CP removal efficiency using T11 in liquid medium was $83 \%$. As biodegradation stimulator riboflavin was added in medium. More than half of initial CP concentration was removed after $24 \mathrm{~h}$. Almost complete removal after 48h. Increase in CP removal efficiency could be attributed to enhanced bacterial growth after riboflavin addition. Two metabolites 3, 5, 6-trichloro-2-pyridinol (TCP) and 3, 5, 6-trichloro-2methoxypyridine (TMP) were detected. Phytotoxicity assay revealed reduction in toxicity of $\mathrm{CP}$. It could be concluded that addition of riboflavin can enhance degradation of $\mathrm{CP}$ and significant reduction in toxicity could be achieved. Mechanism of riboflavin action requires further investigation.
\end{abstract}

Keywords---Biodegradation, Chlorpyrifos, Phytotoxicity, Riboflavin

\section{INTRODUCTION}

C YHLORPYRIFOS (CP) is an organophosphate pesticide. CP is used to control agricultural and house hold pests. Increasing pesticides use has led to an increase in their environmental concentration.

They are known to be toxic for all living organisms. Pesticide elimination from the environment is the need of day. Biodegradation could be one of the possible options for removal of $\mathrm{CP}$. Biodegradation of $\mathrm{CP}$ has been reported using various bacterial strains such as Bacillus pumilus, Sphingobacterium sp. and Pseudomonas sp. [1] , [2] , [3]. In our previous studies we reported biodegradation of $\mathrm{CP}$ using Psychrobacter alimentarius and Pseudomonas kilonensis [4, 5].Biodegradation potential of bacterial strains could be stimulated using various cosubstrates or micro nutrients. Some studies reported effect of toluene, benzene, glucose, lactate, yeast extract, copper, calcium and vitamin addition on degradation of different organic compounds [1] , [5] , [6] , [7]. However to our knowledge effect of vitamin addition on biodegradation of pesticides in general and chlorpyrifos specifically has not been reported yet. Therefore current study was planned with the aim to isolate bacteria capable of $\mathrm{CP}$

Saira Khalid is associated with Institute of Environmental Sciences and Engineering, School of Civil and Environmental Engineering, National University of Sciences and Technology, Islamabad 44000, Pakistan )

Imran Hashmi is associated with Institute of Environmental Sciences and Engineering, School of Civil and Environmental Engineering, National University of Sciences and Technology, Islamabad 44000, Pakistan biodegradation. Bacterial strain was used for biodegradation of $\mathrm{CP}$ in mineral salt medium (MSM).Stimulatory effect of riboflavin addition on biodegradation potential of bacterial strain was also studied. Metabolites formed after $\mathrm{CP}$ biodegradation were detected using GC with electron capture detector. Biodegradation kinetics was also evaluated. Reduction in toxicity level of bioremediated effluents were demonstrated using phytotoxicity assay.

\section{MATERIAL AND METHODS}

\section{A. Isolation of Chlorpyrifos Degrading Bacteria}

Pesticide contaminated agricultural soil samples were collected from district Chakwal (32.55 $\mathrm{N} 72.51^{\circ} \mathrm{E}$.), Punjab, Pakistan. These soil samples were used for isolation of CP degrading bacterial strains. $10 \mathrm{~g}$ of soil was added to mineral salt medium supplemented with selected pesticide. It was incubated at $35^{\circ} \mathrm{C}$ for a week. Sample $(10 \mathrm{ml})$ was then transferred to freshly prepared MSM. This process was repeated thrice. After serial dilution and single colony streaking pure colonies were obtained.

\section{B. 16S rRNA Analysis}

After screening process bacterial strain T11 was selected for further biodegradation studies. T11 was identified using 16S rRNA nucleotide sequence analysis. Briefly DNA was extracted instance matrix (Bio-Rad USA). PCR primers were $27 \mathrm{~F}$ and 1492R. After PCR amplification obtained products were purified and sequenced using big dye terminator cycle sequencing kit (Applied Biosystems, USA).Obtained sequences were compared with sequences reported at NCBI. Phylogenetic analysis was carried out using MEGA4.

\section{Effect of CP on Growth behavior of T11}

Growth of bacterial strain T11 was examined in $30 \mathrm{ml}$ of MSM having $\mathrm{CP}\left(50 \mathrm{mgL}^{-1}\right)$. $\mathrm{pH}$ of medium was adjusted to 7 and inoculated with T11 at initial OD600 $\mathrm{nm}=2$. There were two treatments one with addition of riboflavin and other without its addition. MSM was inoculated with T11 to initial optical density of inoculum was 1 at $600 \mathrm{~nm}$. Flasks were incubated at $35^{\circ} \mathrm{C}$ for 48 hours. Growth was monitored regularly in terms of absorbance at $600 \mathrm{~nm}$.

\section{Biodegradation of Chlorpyrifos and Detection of Metabolites}

For Biodegradation experiments mineral salt medium was prepared and $\mathrm{pH}$ was adjusted to 7.CP was added at $50 \mathrm{mgL}^{-1}$ and inoculated with $\mathrm{T} 11\left(\mathrm{OD}_{600 \mathrm{~nm}}=2\right)$. It was incubated a $35^{\circ} \mathrm{C}$ for 48hours.Samples were collected regularly and analyzed for remaining $\mathrm{CP}$ concentration, optical density and metabolites. 
Bacterial growth in terms of absorbance at $600 \mathrm{~nm}$ was measured using spectrophotometer (model). CP was measured using GC ECD. For sample extraction ethyl acetate was used. For metabolites acetone was used for sample extraction. GCECD conditions are mentioned in [4], [5] were followed. Reusability of T11 culture was tested by cyclic use. After completion of one cycle $\mathrm{CP}$ was again added in same medium without any nutrient or bacterial addition. Samples were collected at start and end of the experiment and analyzed for remaining $\mathrm{CP}$ concentration.

\section{E. Phytotoxicity Assay}

To evaluate the toxicity level of treated effluents a phytotoxicity test was employed. For the seeds of a common agricultural crop triticum aestivum (wheat) were used following method of Saez [8]. Briefly filter papers were placed in sterilized glass petri plates. 20 seeds were placed in each petri plate and treated with $5 \mathrm{ml}$ of tap water (positive control), abiotic control (negative control) and biotreated effluents with and without riboflavin addition. Germinated seeds were counted and germination \% was calculated. At the end root and shoot length was also measured using $\mathrm{cm}$ scale. Vigour index was calculated using following (1)

$$
\text { Vigour index }(V I)=(L R+L T) * \frac{G}{10}
$$

\section{RESULTS AND DISCUSSION}

\section{A. Identification and Growth of Alcaligens sp. T11}

Strain T11 was identified as Alcaligens sp. NCBI GenBank accession number for T11 is KT037096. T11 was able to grow on varying $\mathrm{pH}$ and temperature. Growth of T11 in MSM supplemented with $\mathrm{CP}$ was monitored with and without addition of riboflavin. At any given time period throughout the incubation time growth of T11 was higher in medium with riboflavin than without Riboflavin addition. At 24 hour growth with and without riboflavin was 1.45 and 1.38 (OD at $600 \mathrm{~nm}$ ). A drop in growth was observed at 30 and 36 hours with and without riboflavin addition respectively (Fig. 1). At 48 h 1.17 and $0.99(\mathrm{OD}$ at $600 \mathrm{~nm})$ was observed in medium with and without CP addition (Fig. 1). In system without CP but with riboflavin no increase in growth was observed. Reason for this could be unavailability of carbon source. From these results it could be concluded that Riboflavin has positively influenced bacterial growth in the presence of CP. Enhanced growth of oil degrading bacterial strains was observed in the presence of vitamin as compared to medium without it [9]. Adding vitamins B complex has improved growth of fungal strain in media containing phenol [7].

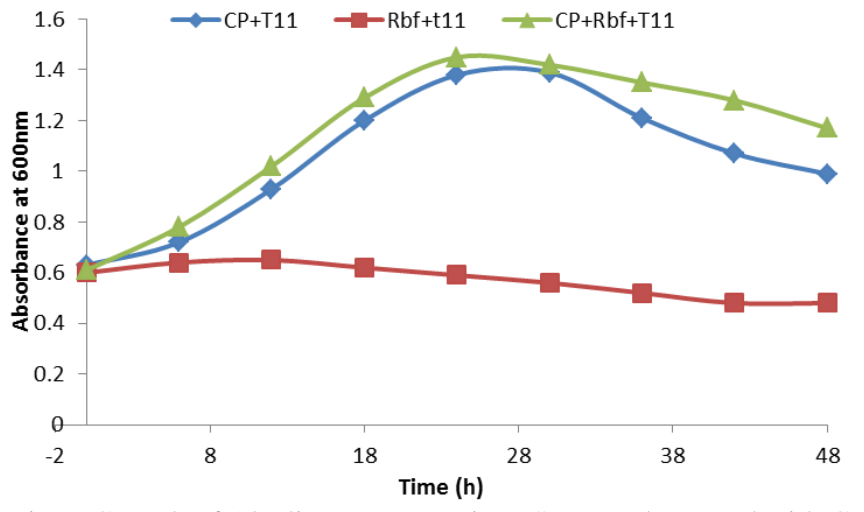

Fig 1. Growth of Alcaligens sp. T11 in MSM supplemented with CP as sole carbon source with and without addition of riboflavin.

\section{B. CP Degradation and Generation of Metabolites}

Fig. 2 depicts concentration of CP from 0 upto $48 \mathrm{~h}$. After $12 \mathrm{~h}$ of incubation $\mathrm{CP}$ concentration was dropped to 30.2 and $41 \%$ without riboflavin and with riboflavin addition. after one half of incubation time (24h)82\% CP removal was detected in sample with riboflavin addition whereas only $56.8 \%$ removal without riboflavin. At the end of incubation period (48h),CP removal efficiency was 98 and $83.74 \%$ in system with and without riboflavin addition respectively(Fig.2a). Experimental data fits best to first order kinetic model $\left(R^{2}>0.98\right)$. Addition of riboflavin enhanced degradation rate from 0.03 to $0.08\left(\mathrm{~h}^{-1}\right)$ (Fig. 2b).

Drop in concentration of $\mathrm{CP}$ with the passage of time means it was broken down into some other intermediate compounds. For detection of intermediate compounds or metabolites samples were collected and analyzed. Initially TCP was not detected in any system; however after $6 \mathrm{~h}$ of incubation 2.3 and $0.6 \mathrm{mgL}^{-1} \mathrm{TCP}$ was detected in system with and without CP addition respectively (Fig. 3a). Maximum concentration of TCP was detected in system with Riboflavin addition at $24 \mathrm{~h}$ i.e. $15.3 \mathrm{mgL}^{-1}$. After that a drop in TCP concentration was observed (Fig. 3a). This decrease in TCP concentration means that it was also biodegraded into some other metabolite. Most common metabolite reported in literature is TMP [4]. 0.65 $\mathrm{mgL}^{-1}$ TMP was detected in system without riboflavin after 12 hours of incubation and maximum concentration was observed at $40 \mathrm{~h}$. After 6 hours $0.95 \mathrm{mg} / \mathrm{L}^{-1}$ TMP was detected with Riboflavin addition (Fig. 3b). Maximum concentration of TMP $9.21 \mathrm{mgL}^{-1}$ was observed at $30 \mathrm{~h}$ after which a drop in concentration was observed (Fig. 3b). TMP is water soluble, less toxic and less persistent than TCP. After certain time period a drop in TMP concentration was observed. This means TMP was further degraded into natural compounds or eater soluble products. With Addition of Riboflavin almost complete removal of $\mathrm{CP}$ was achieved this could be the result of enhanced microbial growth. Exact mechanism behind this is still unknown and needs to be investigated. Possible degradation pathway was ;

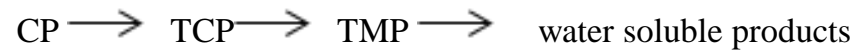



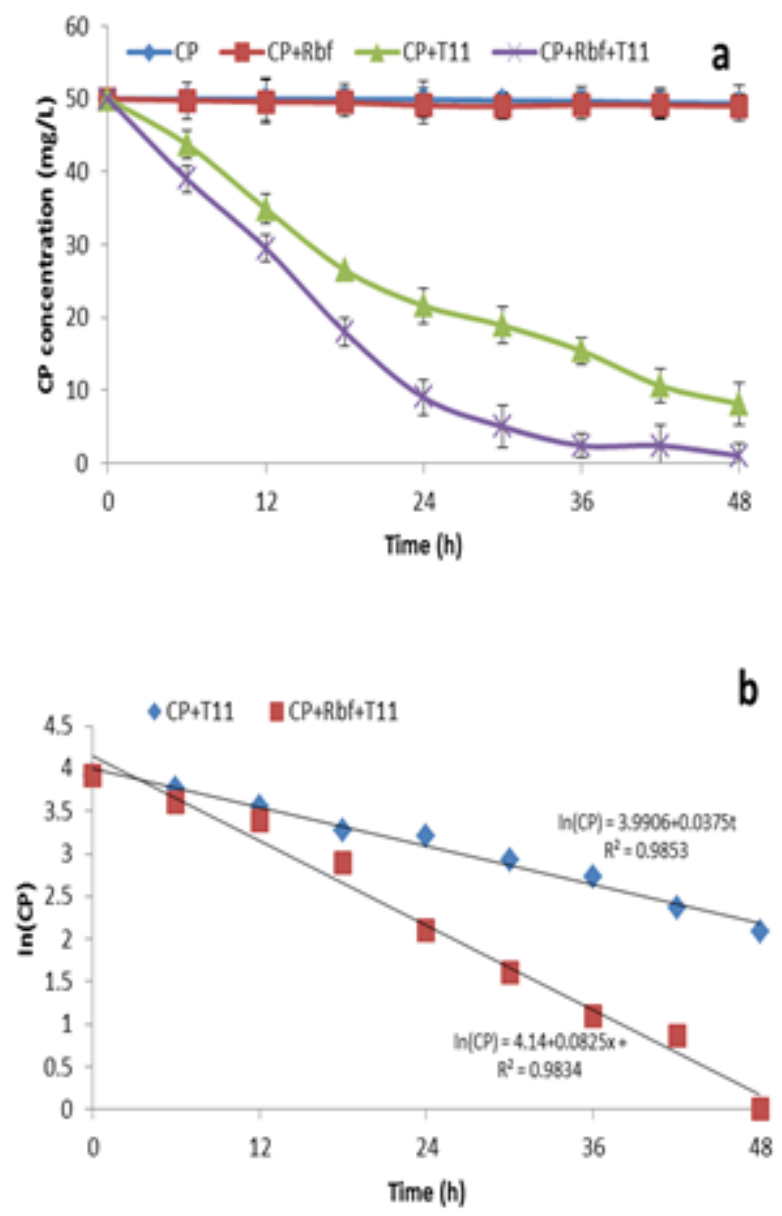

Fig. 2. Biodegradation of chlorpyrifos using Alcaligens sp. T11 in flask culture with and without addition of riboflavin (a) concentration of $\mathrm{CP}$ with time (b) relationship of $\operatorname{In}(\mathrm{CP})$ with time.

Some studies reported enhanced photodegradation of organic compounds in the presence of riboflavin. For this purpose medium with CP and Riboflavin but without addition of T11 was used as control. No considerable degradation was observed in control. From these results it could be concluded that reason for enhanced $\mathrm{CP}$ degradation in the presence of riboflavin was due to stimulated bacterial activity. Adding vitamin solution having riboflavin improved biodegradation of phenol by $16 \%$ [10]. Vitamin addition enhanced degradation of phenanthrene in soil [6].
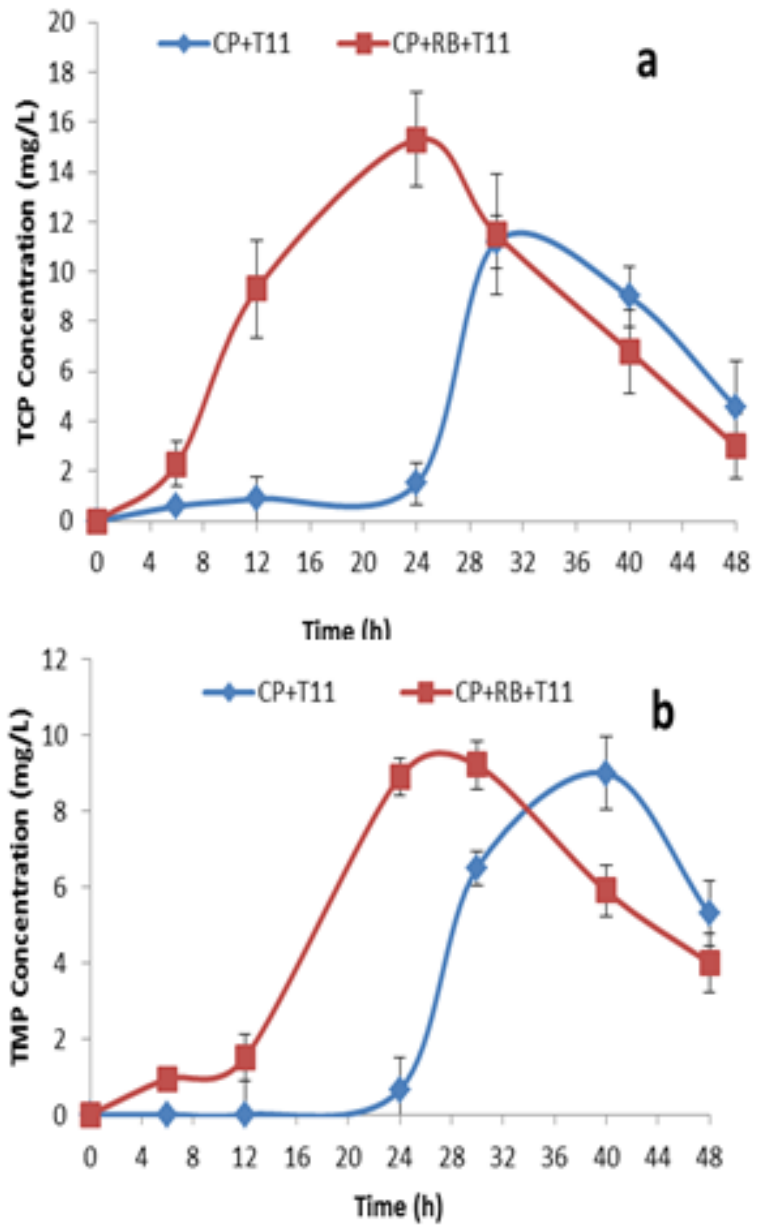

Fig. 3. Formation of metabolites after CP degradation (d) TCP concentration with time (b) TMP concentration with time.

\section{Phytotoxicity Study}

Seeds of triticum aestivum were irrigated with tap water (T1), abiotic control (T2) and biotreated effluents without riboflavin (T3) and biotreated effluents with riboflavin (T4) effluents. Germination \% with T1, T2, T3 and T4 was 95, 55, $70,80 \%$ (Fig. 4a). Root length and shoot length with T1 was 7.83 and 10.4. Root with T3 and T4 was 4.55 and 5.89. Shoot length with T3 and T4 was 5.86 and 7.81(Fig. 4a). Root and shoot length with T2 was 2.03 and $2.56 \mathrm{~cm}$. Vigor index is an indicator of seedling health. Vigor index was significantly higher with T3 and T4 as compared to abiotic control (T2) (Fig. 4b). This indicated significant decrease in toxicity of CP after microbial breakdown. This confirms potential of T11 to cause a significant decrease in toxicity of $\mathrm{CP}$. 

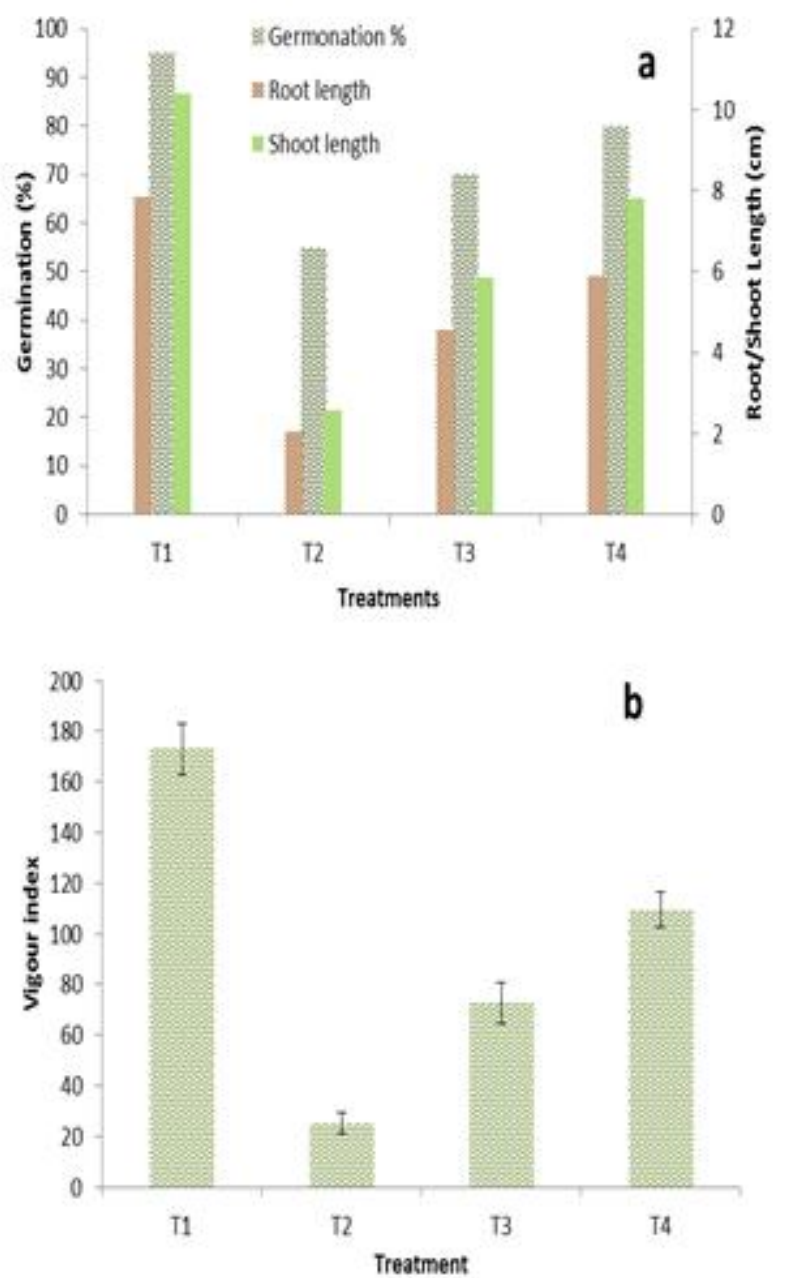

Fig. 4 Growth of triticum aestivum after treatment with tap water, treated and untreated effluents (a) germination $\%$ and root, shoot length (b) vigour index with all treatments.

\section{Reuse of Bacterial Culture}

With increase in cycle a gradual increase in CP removal efficiency was observed. Possibly, because bacterial cells have developed tolerance to $\mathrm{CP}$ with each cycle. From first cycle a gradual increase in CP removal efficiency was observed upto $7^{\text {th }}$ cycle i.e. $99 \%$ (Fig. 5). At $10^{\text {th }}$ cycle removal efficiency was $98 \%$ this means more cycles could be performed. This is very beneficial for field scale application as T11 was able to maintain a sustainable population over a long period of time. In our previous studied CP degradation using Pseudomonas kilonensis SRK1 for 6 cycles in sequencing batch reactor [5]. Raoultella planticola was used for 10 cycles for atrazine metabolism [11].

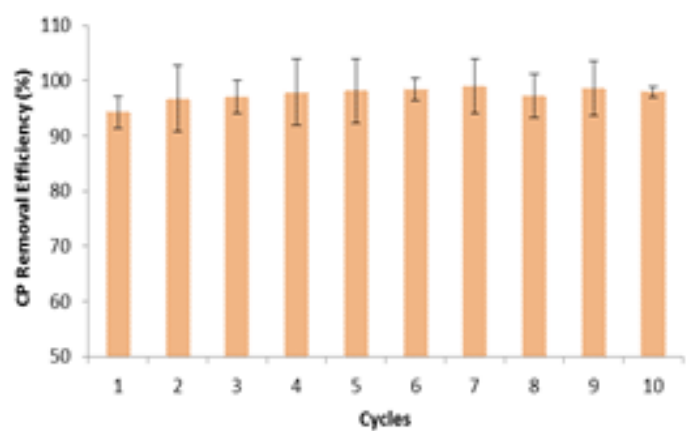

Fig.5. CP removal efficiency in mineral slat medium for 10 cycles (initial CP concentration $50 \mathrm{mg} / \mathrm{L}$, incubation time; 48hour, inoculum; T11, inoculum density at $600 \mathrm{~nm} ; 2$ )

\section{REFERENCES}

[1] S. Anwar, F. Liaquat, Q.M. Khan, Z.M. Khalid, S. Iqbal, 2009. "Biodegradation of chlorpyrifos and its hydrolysis product3, 5, 6trichloro-2-pyridinol by Bacillus pumilus strain C2A1" Journal of Hazard. Mat, vol. 168, 2009, pp. 400-405. http://dx.doi.org/10.1016/j.jhazmat.2009.02.059

[2] M. Yadav, N. Srivastva, S.R. Singh, S.N. Upadhyay, S.K. Dubey, "Biodegradation of chlorpyrifos by Pseudomonas sp. in a continuous packed bed bioreactor", Bioresource Technol. vol. 165, 2014, pp. 265269. http://dx.doi.org/10.1016/j.biortech.2014.01.098

[3] J. Abraham, S. Silambarasan, "Biodegradation of chlorpyrifos and its hydrolyzing metabolite 3,5,6trichloro-2-pyridinol by Sphingobacterium sp. JAS3”. Process Biochemistry, vol. 48, 2013, pp. 1559-1564. http://dx.doi.org/10.1016/j.procbio.2013.06.034

[4] S. Khalid, I. Hashmi, "Biotreatment of chlorpyrifos in a bench scale bioreactor using Psychrobacter alimentarius T14", Environ. Technolo. 2015 DOI:10.1080/09593330.2015.1069406. http://dx.doi.org/10.1080/09593330.2015.1069406

[5] Khalid, S., Hashmi, I., Khan, S.J. "Bacterial Assisted Degradation of Chlorpyrifos: The Key Role of Environmental Conditions, Trace Metals and Organic Solvents" (Acccepted for publication)," J. of Enviro. Management, to be published. 2015. 10.1016/j.jenvman.2015.11.030. http://dx.doi.org/10.1016/j.jenvman.2015.11.030

[6] A.P. Jonsson, T.L. Östberg, "The effects of carbon sources and micronutrients in whey and fermented whey on the kinetics of phenanthrene biodegradation in diesel contaminated soil" Journal of Hazardous Materials, vol. 192, 2011, pp. 1171-1177. http://dx.doi.org/10.1016/j.jhazmat.2011.06.024

[7] E.H.F. Abd El-Zaher, Y.A.G. Mahmoud, M.M. Aly, "Effect of different concentrations of phenol on growth of some fungi isolated from contaminated soil" African Journal of Biotechnology. vol. 10, 2011, pp.384-1392.

[8] J.M. Saez, A. Alvarez, C.S. Benimeli, M.J. Amoroso, "Enhanced Lindane removal from soil slurry by immobilized Streptomyces consortium", Int Biodeter. \& Biodeg., vol. 93, 2014, pp. 63-69.

[9] S.S. Radwan, A.S. AI-Muteirie, "Vitamin requirements of hydrocarbonutilizing soil bacteria", Microbiol. Res.vol.155,2001,pp. 301-307. http://dx.doi.org/10.1016/S0944-5013(01)80008-2

[10] D. Kafkewitz, F. Fava, P.M. Armenante, " Effect for vitamins on the aerobic degradation of 2-chlorophenol, 4-chlorophenil, and 4chlorobiphenyl" Applied Microbiology and Biotechnology, vol. 46, 1996, pp. 414-421. http://dx.doi.org/10.1007/BF00166239

[11] N. Swissa, Y. Nitzan, Y. Langzam, R. Cahan, “ Atrazine biodegradation by a monoculture of Raoultella planticola isolated from a herbicides wastewater treatment facility", Int Biodeter Biodegr., vol.92,2014, pp. 92, 6-11. 